\title{
STUDY OF STRUCTURAL AND DIELECTRIC PROPERTIES OF PZT CERAMICS MODIFIED BY SM ION SUBSTITUTION
}

\author{
Kanay Barik $^{1}$, Rajiv Kumar ${ }^{2}$, Sanjay Kumar Sinha ${ }^{3}$ \\ ${ }^{1}$ Research Scholar, Kolhan University, India. Email- kanaybarik2010@gmail.com \\ ${ }^{2}$ Jamshedpur Workers College, Jamshedpur 831012, India. Email-drrajiv96@gmail.com \\ ${ }^{3}$ Associate Professor, BIT Mesra, Patna, India. Email-sksinha@bitmesra.ac.in
}

\begin{abstract}
The Effect of Sm substitution on the structural and dielectric properties of $P b_{1-x} S m_{x}\left(Z r_{0.4} T i_{0.6}\right)_{1-x / 4} O_{3}(P S Z T)(x=0.00,0.04$, 0.08 and 0.12) composition prepared from mixed oxide method at high temperature were synthesized. The formation of single phase compounds were confirmed by XRD studies which were found to be in tetragonal phase at room temperature The dielectric constant, tangent loss, and transition temperature of PSZT as a function of temperature at selected frequencies has exhibited that maximum or peak are strongly dependent on Sm content. When the temperature of PSZT samples is increased above transition temperature, dielectric constant begins to decrease obeying Curie - Weiss law. The Conduction process was found to be mixed type. The variation of dielectric constant and tangent loss with temperature at selected frequencies exhibit their phase transition above room temperature.
\end{abstract}

Keywords: Ferro electricity, Perovskite, Diffraction, Transducer, Doping.

\section{INTRODUCTION}

There has been considerable interest in the solid solutions of lead zirconate-titanate, due to its possible forefront applications in the areas of research as well as in industrial applications [1-3]. The properties of PZT are very much sensitive to its compositional fluctuations near the morphotropic phase boundary (MPB), particle size, doping, calcinations and sintering temperature. PZT is used in a wide range of piezoelectric, pyroelectric and ferroelectric device application A considerable amount of works have been done on modified PZT ceramics prepared from hightemperature solid-state reaction technique. Now days, with suitable modification in the compound, it is widely used for actuators, pyroelectric detectors, transducers, electro - optic, ferroelectric random access memory, sensors, etc. The physical properties and device parameters of PZT - based compounds are greatly influenced by chemical substitutions, synthesis process, and some other factors. It is well observed that the La-modified PZT has tremendous applications in electronics and electro-optics [4]. The literature survey on pure and modified PZT materials reveals that no systematic studies have been reported on physical properties and device parameters of Sm-substituted PZT (i.e., PSZT) with $\mathrm{Zr} / \mathrm{Ti}$ ratio 40/60 [5-7]. In view of the above, we have studied the effect of samarium substitution on structural, dielectric, properties ceramics, which is reported here.

\section{Experimental Details}

The samples of PSZT i.e. $\mathrm{Pb}_{1-\mathrm{x}} \mathrm{Sm}_{\mathrm{x}}\left(\mathrm{Zr}_{0.4} \mathrm{Ti}_{0.6}\right)_{1-\mathrm{x} / 4} \mathrm{O}_{3}$ (where $x=0.00,0.04,0.08$, and 0.12 ) were prepared by a high- temperature solid-state reaction technique. In an alumina crucible, the homogeneous mixed ingredients were calcined at an optimized temperature of $1100^{\circ} \mathrm{C}$ for 10 hours. Using hydraulic press, the calcined powders, with small amount of polyvinyl alcohol as binder, were converted into pellets at a pressure of $4 \times 10^{6} \mathrm{~N} / \mathrm{m}^{2}$. These pellets were sintered in an alumina crucible at an optimized temperature of $1200^{\circ} \mathrm{C}$ for 10 hours aiming to get nearly $97 \%$ of theoretical density. The X-ray diffraction (XRD) data on the calcined powders were recorded using X-ray diffractometer (Rigaku Miniflex, Japan) with $\lambda=1.5405^{\circ} \mathrm{A}$ in a wide range of Bragg's angles $2 \theta\left(20^{\circ} \leq 2 \theta \leq 80^{\circ}\right)$ at a scanning rate of $3 \% /$ minute. The dielectric data of the materials were obtained on silver electroded samples using phase sensitive multimeter in a wide range of frequency $\left(10^{2}-10^{6} \mathrm{~Hz}\right)$ and temperature (room temperature $-500{ }^{\circ} \mathrm{C}$ ) at a potential difference of $1 \mathrm{~V}$ with the stabilized temperature at an interval of $2.5^{\circ} \mathrm{C}$.

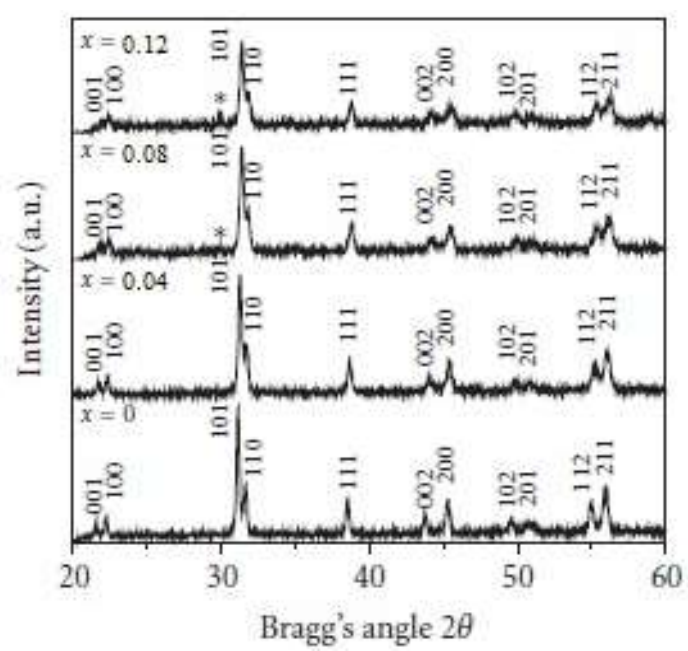

Figure 1: The comparison of XRD patterns of $\mathrm{Pb}_{1-\mathrm{x}} \mathrm{Sm}_{\mathrm{x}}\left(\mathrm{Zr}_{0.4} \mathrm{Ti}_{0.6}\right)_{1-\mathrm{x} / 4} \mathrm{O}_{3}$ (for $x=0.00,0.04,0.08$, and 0.12 ). 


\section{Results and Discussion}

3.1 Structural Analysis - The nature of XRD patterns of $\mathrm{Pb}_{1-\mathrm{x}} \mathrm{Sm}_{\mathrm{x}}\left(\mathrm{Zr}_{0.4} \mathrm{Ti}_{0.6}\right)_{1-\mathrm{x} / 4} \mathrm{O}_{3}$ (PSZT) with $x=0.00,0.04$, 0.08 , and 0.12 (Figure 1) confirms the formation of single phase with tetragonal crystal structure [8]. Using computer software POWDMULT all the reflection peaks were indexed in tetragonal crystal system. On the basis of best agreement between the observed (obs) and the calculated (cal) $d$ - spacing (i.e., $\sum \Delta d=d_{\mathrm{obs}}-d_{\text {cal }}=$ minimum), all the PSZT compounds were found to be in tetragonal crystal system with their refined lattice parameters given in Table 1. In the XRD patterns, there is an additional peak (for $x \geq$ 0.08 ) usually referred as secondary or pyrochlore phase $[9$, 10]. Though these peaks are undesirable, it is some time essential for formation of the perovskites [11]. The percentage of pyrochlore phase in PSZT for $x=0.08$ and 0.12 was estimated as $6 \%$ and $8.5 \%$, respectively.

3.2 Dielectric Study - The variation of relative dielectric constant $\left(\varepsilon_{\mathrm{r}}\right)$ of PSZT having Sm contents $x=0.00,0.04$, 0.08 , and 0.12 with temperature at selected frequencies $\left(10^{3}-10^{6} \mathrm{~Hz}\right)$ is shown in Figure 2 below. It is found that $\varepsilon_{\mathrm{r}}$ decreases on increasing frequency which indicates a normal behavior of the dielectric materials. The higher values of $\varepsilon_{\mathrm{r}}$ at lower frequency are due to the simultaneous presence of all types of polarizations (space charge, dipolar, ionic, electronic, etc.) which is found to decrease with the increase in frequency. At high frequencies $\left(>10^{12} \mathrm{~Hz}\right)$ electronic
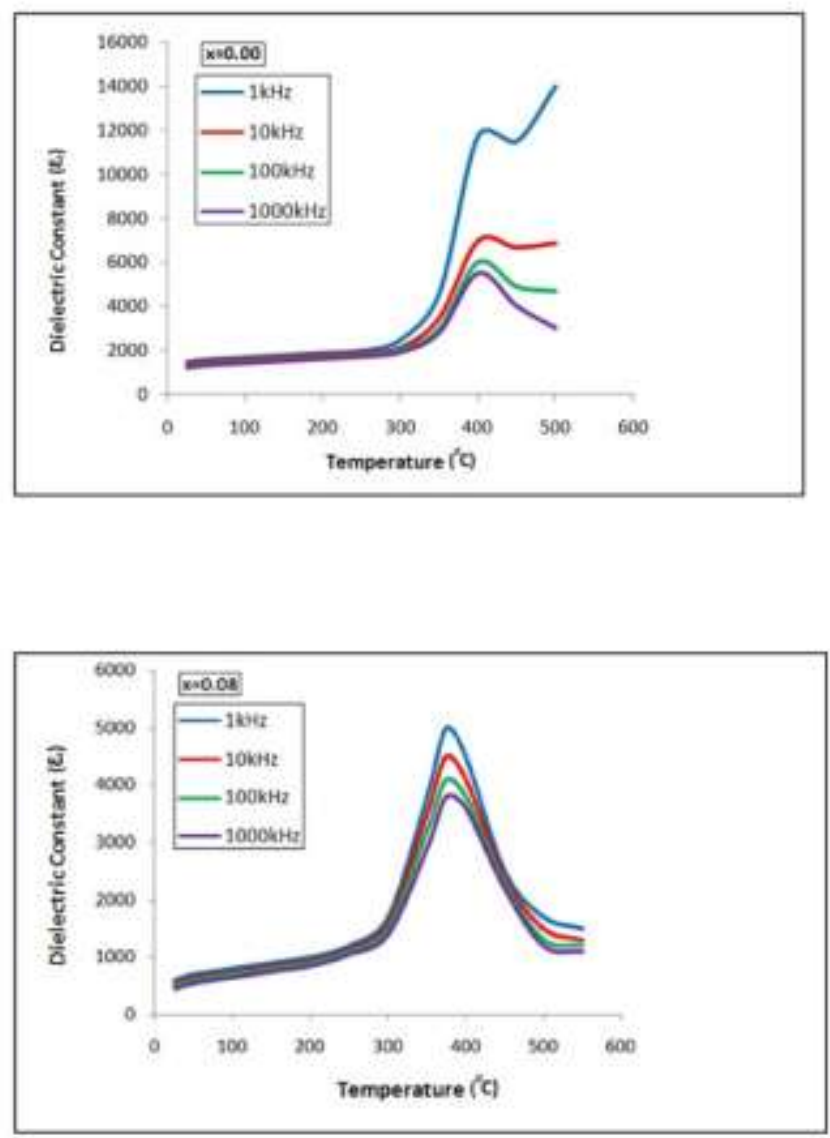

polarization only exists in the materials. When temperature of PSZT samples is increased, $\varepsilon_{\mathrm{r}}$ first increases slowly and then rapidly up to a maximum value. Temperature of the material corresponding to $\varepsilon_{\max }$ is called Curie or critical temperature $\left(\mathrm{T}_{\mathrm{c}}\right)$. As at this $\mathrm{T}_{\mathrm{c}}$, phase transition takes place between ferroelectric-pyroelectric phases so it is also called transition temperature. At the higher temperature $\left(\geq T_{c}\right)$, the space charge polarization originates due to mobility of ions and imperfections in materials and thus contributes to a sharp increase in $\varepsilon_{\mathrm{r}}$ [12]. The value of $\varepsilon_{\max }$ is found to be highest for PZT. As Sm content in PSZT increases, the value of $\varepsilon$ max exhibits a sharp decrease for $\mathrm{x}=0.04$, then an increase for $x=0.08$, and again decrease for $x=0.12$. The value of $T_{c}$ is found to be highest for PZT which decreases gradually on increasing Sm content in PSZT. However, for each PSZT samples Tc is found to be unaffected with the change in frequency supporting the nonrelaxor behavior of Sm-modified PZT. The values of $\varepsilon_{\max }$ and $\mathrm{T}_{\mathrm{c}}$ of PSZT are compared in Table 1.

The frequency-temperature dependence of tangent loss ( $\tan$ $\delta$ ) of PSZT is shown in Figure 2. With the increase in temperature, $\tan \delta$ is found to be very low and almost remains constant up to $\mathrm{T}_{\mathrm{c}}$ beyond which it indicates a significant increase. The nature of variation of $\tan \delta$ at higher frequency and temperature can be explained by space-charge polarization. This $\tan \delta$ decreases with the increase in frequency as expected [13 - 15].
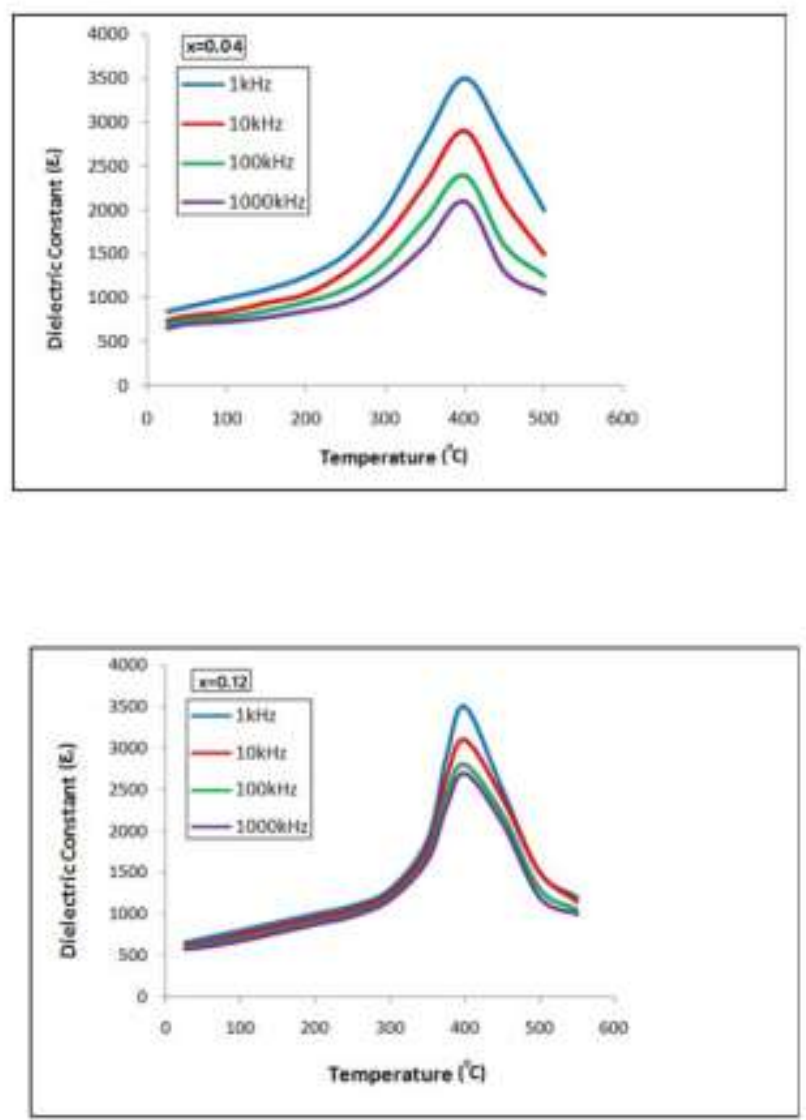

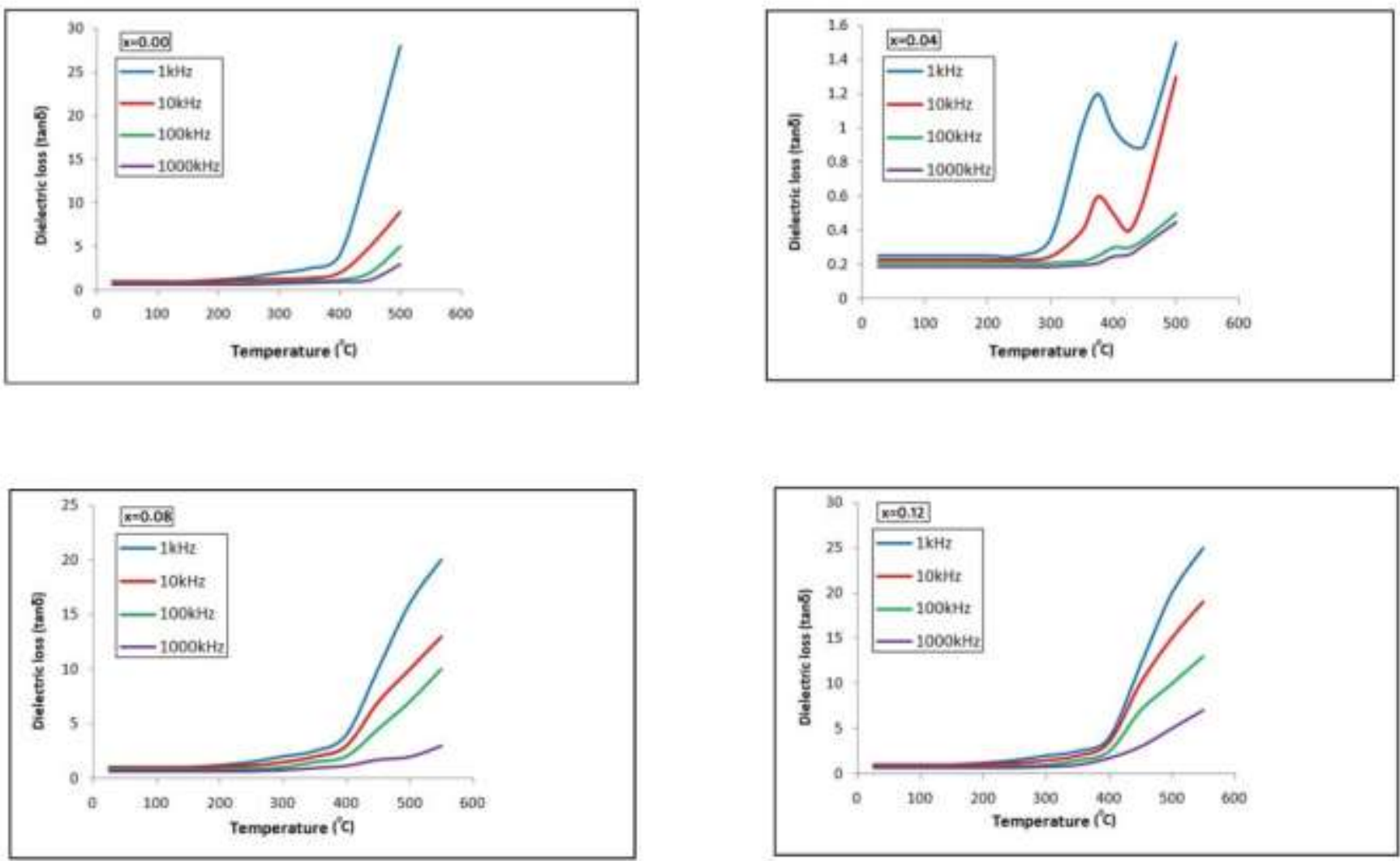

Figure 2: Temperature-frequency dependence of relative dielectric constant $\left(\varepsilon_{\mathrm{r}}\right)$ and $\operatorname{tangent} \operatorname{loss}(\tan \delta)$ $\mathrm{Pb}_{1-\mathrm{x}} \mathrm{Sm}_{\mathrm{x}}\left(\mathrm{Zr}_{0.4} \mathrm{Ti}_{0.6}\right)_{1-\mathrm{x} / 4} \mathrm{O}_{3}$ for $x=0.00,0.04,0.08$, and 0.12

Table 1: Comparison of the lattice parameters, $\varepsilon_{\max }$ and $\mathrm{T}_{\mathrm{c}}$ of $\mathrm{Pb}_{1-\mathrm{x}} \mathrm{Sm}_{\mathrm{x}}\left(\mathrm{Zr}_{0.4} \mathrm{Ti}_{0.6}\right)_{1-\mathrm{x} / 4} \mathrm{O}_{3}$ for $\mathrm{x}=0.00,0.04,0.08$ and 0.12

\begin{tabular}{|lllll|}
\hline \multicolumn{5}{c|}{ Sm composition } \\
Parameters & $\mathrm{x}=0.00$ & $\mathrm{x}=0.04$ & $\mathrm{x}=0.08$ & $\mathrm{x}=0.12$ \\
$\mathrm{a}$ & $4.0042(21)$ & $3.9966(50)$ & $3.9725(50)$ & $3.9785(50)$ \\
$\mathrm{c} / \mathrm{a}$ & $4.1348(21)$ & $4.1146(50)$ & $4.0844(50)$ & $4.1081(50)$ \\
$\varepsilon_{\max }$ & $1.0326(21)$ & $1.02952(50)$ & $1.02816(50)$ & $1.0325(50)$ \\
$\mathrm{T}(\mathrm{C})$ & $404 \pm 0.25$ & $383 \pm 0.25$ & $371 \pm 0.25$ & $364 \pm 0.25$ \\
\hline
\end{tabular}

\section{CONCLUSIONS}

Structural analysis using room temperature $\mathrm{X}$ - ray diffraction data obtained from the calcined powders of polycrystalline samples of Sm-modified PZT (i.e., $\mathrm{Pb}_{1-\mathrm{x}} \mathrm{Sm}_{\mathrm{x}}\left(\mathrm{Zr}_{0.4} \mathrm{Ti}_{0.6}\right)_{1-\mathrm{x} / 4} \mathrm{O}_{3}$ ) has confirmed their tetragonal phase with the presence of a small amount of pyrochlore phase during higher concentration of $\mathrm{Sm}$ (6\% for $x=0.08$ and $8.5 \%$ for 0.12 ). The dielectric constant, tangent loss, and transition temperature of PSZT as a function of temperature at selected frequencies has exhibited that maximum or peak are strongly dependent on Sm content. The electrical conductivity (ac) of PSZT may not only due to singly ionized in low temperature (ferroelectric phase) region but also due to doubly ionized in the hightemperature region.

\section{REFERENCES}

[1]. Buchanan, R. C., Ceramic Materials for Electronics. Marcel Decker, New York, 1986.

[2]. Herbert, J. M., Ceramic Dielectrics and Capacitors. Gordon and Breach Science Publishers, New York, 1985.

[3] G. H. Haertling, "Piezoelectric and electro optic ceramics," in Ceramic Materials for Electronics, R. C. Buchanan, Ed., chapter 3, pp. 139-225, Marcel Dekker, New York, NY, USA, 1991.

[4] B.-S. Chiou, J. N. Kuo, and H. T. Dai, "The preparation of PLZT ceramics from a sol-gel process," Journal of Electronic Materials, vol. 19, no. 4, pp. 393-397, 1990.

[5] U. Chon, K.-B. Kim, H. M. Jang, and G.-C. Yi, "Fatigue-free samarium-modified bismuth titanate film capacitors having large spontaneous polarizations," Applied Physics Letters, vol. 79, no. 19, pp. 3137-3139, 2001. 
[6] S. K. Pandey, O. P. Thakur, D. K. Bhattacharya, C. Prakash, and R. Chatterjee, "Structural and electrical properties of Sm3+ substituted PZT ceramics," Journal of Alloys and Compounds, vol. 468, no. 1-2, pp. 356-359, 2009.

[7] S. R. Shannigrahi and R. N. P. Choudhary, "Structural and electrical properties of sol-gel prepared Sm modified ceramics," British Ceramic Transactions, vol. 101, no. 1, pp. 25-29, 2002.

[8] C. Prakash and J. K. Juneja, "Investigations on Sm- and Nb substituted PZT ceramics," Modern Physics Letters B, vol. 20, no. 29, pp. 1879-1882, 2006.

[9] A. K. Tripathi, T. C. Goel, and C. Prakash, "Preparation of 4:55:45 samarium doped PZT films by sol-gel technique and their characterization," Materials Science and Engineering B, vol. 96, no. 1, pp. 19-23, 2002.

[10] R. Khazanchi, S. Sharma, and T. C. Goel, "Effect of rare earth Europium substitution on themicrostructure, dielectric, ferroelectric and pyroelectric properties of PZT ceramics," Journal of Electro ceramics, vol. 14, no. 2, pp. 113-118, 2005.

[11] J. Lian, L.Wang, J. Chen, et al., "The order-disorder transition in ion-irradiated pyrochlore," Acta Materialia, vol. 51, no. 5, pp. 1493-1502, 2013.

[12] S. R. Shannigrahi, F. E. H. Tay, K. Yao, and R. N. P. Choudhary, "Effect of rare earth (La, Nd, Sm, Eu, Gd, Dy, $\mathrm{Er}$ and $\mathrm{Yb}$ ) ion substitutions on the micro structural and electrical properties of sol-gel grown PZT ceramics," Journal of the European Ceramic Society, vol. 24, no. 1, pp. 163-170, 2004.

[13] C. Ang, Z. Yu, and L. E. Cross, "Oxygen-vacancyrelated low frequency dielectric relaxation and electrical conduction in Bi: $\mathrm{SrTiO}_{3}$," Physical Review B, vol. 62, no. 1, pp. 228-236, 2000.

[14].Rajiv Kumar, Ph. D Thesis, Ranchi University (1994)

[15].D.K Mahato, R.K. Choudhary, S.C.Srivastava, J. Appl.

Sci.,6 (2006)716 